\title{
Uptake, accumulation, translocation and subcellular distribution of perchlorate in tea (Camellia sinensis $L$.) plants
}

Yabo Liang ${ }^{\dagger, \S}$, Li Zhou ${ }^{\dagger, \#, ~ X i n z h o n g ~ Z h a n g ~}{ }^{\dagger, \#, ~ H u a n ~ Y u ̛ ~}{ }^{\dagger, \S}$, Mingming Guo ${ }^{\dagger, \S}$, Jiawei Yu $^{\dagger, \S}$, Xinru Wang ${ }^{\dagger, \#}$, Mei Yang ${ }^{\dagger, \#}$, Zhengyun Lou $^{\dagger, \#}$, Fengjian Luo ${ }^{\dagger, \#}$, Hezhi Sun, ${ }^{*},, \#$ Zongmao Chen ${ }^{*}, ;, \#$

$\dagger$ Tea Research Institute, Chinese Academy of Agricultural Sciences, Hangzhou 310008, China

${ }^{\S}$ Graduate School of Chinese Academy of Agricultural Sciences, Beijing 100081, China

\#Key Laboratory of Tea Quality and Safety Control, Ministry of Agriculture and Rural Affairs, Hangzhou 310008, China

\section{* Corresponding authors:}

Hezhi Sun, sunhezhi@tricaas.com.

Tel: +86 571-86653171; fax: +86 571-86650100

Zongmao Chen, zmchen2006@163.com.

Tel: +86 571-86650100; fax: +86 571-86650100 


\section{Table of Contents:}

\section{MATERIALS AND METHODS}

\section{Instruments}

Table S1. Nutrient solution composition.

Table S2. Spiked levels, recoveries, relative standard deviations (RSDs).

Table S3. Linear regression, $\mathrm{R}^{2}$, MEs.

Table S4. Linear regression of tissue perchlorate concentration (21 th day) and exposure concentration.

Table S5. The bioconcentration factor (BCF) of field samples.

Table S6. The BCFs of perchlorate in plant leaves.

Figure S1. Perchlorate concentration in root of Wuniuzao under 1 and $10 \mathrm{mg} / \mathrm{L}$ perchlorate treatment as a function of exposure time. $(n=3)$

Figure S2. Perchlorate concentration of different tissues in each subcellular fraction. Roots(R), new shoots(B), mature leaves(M). Fuding Dabaicha(D), Wuniuzao(W). Subcellular fraction: cell wall(F1), cell organelles(F2) and soluble fractions(F3). (n=3) 


\section{MATERIALS AND METHODS}

\section{Instruments.}

Quantitative analysis of perchlorate was determined by a Waters ACQUITY UPLC H-Class coupled to a Waters Xevo TQ-S Micro triple-quadrupole mass spectrometer. Perchlorate was separated using a Thermo Hypercarb column $(2.1 \times 100 \mathrm{~mm}, 5 \mu \mathrm{m})$ with the column temperature at $35^{\circ} \mathrm{C}$. The mobile phase consisted of acetonitrile (A) and $10 \mathrm{mM}$ ammonium acetate in water (B) was applied at a flow rate of $0.3 \mathrm{~mL} / \mathrm{min}$ with the following gradient program:0-2 $\mathrm{min}$ at $50 \% \mathrm{~A}, 2-2.5 \mathrm{~min}$ at $50-90 \% \mathrm{~A}, 2.5-3.5$ $\min$ at $90 \% \mathrm{~A}, 3.5-4 \mathrm{~min}$ at $90-50 \% \mathrm{~A}, 4-5 \mathrm{~min}$ at $50 \% \mathrm{~A}$. The injection volume was 5 $\mu \mathrm{L}$.

Waters Xevo TQ-S Micro was operated in negative ionization mode (ESI-). The source temperature was $150^{\circ} \mathrm{C}$. Capillary voltage and cone voltage were $0.2 \mathrm{kV}$ and 10 $\mathrm{V}$, respectively. The desolvation temperature was $350^{\circ} \mathrm{C}$. Source gas $\left(\mathrm{N}_{2}, 99.5 \%\right)$ was at the flow rate of $650 \mathrm{~L} / \mathrm{H}$ for desolvation and $50 \mathrm{~L} / \mathrm{H}$ for cone. In the multiple reaction monitoring (MRM) mode, the dwell time was $0.082 \mathrm{~s}$. Collision gas (Ar, 99.995\%) was at the flow rate of $0.25 \mathrm{~mL} / \mathrm{min}$. Cone voltage and collision voltage were $16 \mathrm{~V}$ and 18 $\mathrm{V}$, respectively. 
Table S1. Nutrient solution composition.

\begin{tabular}{cccc}
\hline Nutrient & $\begin{array}{c}\text { Concentration } \\
(\mathrm{mmol} / \mathrm{L})\end{array}$ & Nutrient & $\begin{array}{c}\text { Concentration } \\
(\mu \mathrm{mol} / \mathrm{L})\end{array}$ \\
\hline$\left(\mathrm{NH}_{4}\right)_{2} \mathrm{SO}_{4}$ & 0.60 & $\mathrm{EDTA} \mathrm{Na}_{2} \mathrm{Fe}$ & 4.20 \\
$\mathrm{Ca}\left(\mathrm{NO}_{3}\right)_{2}$ & 0.30 & $\mathrm{H}_{3} \mathrm{BO}_{3}$ & 7.00 \\
$\mathrm{KH}_{2} \mathrm{PO}_{4}$ & 0.07 & $\mathrm{MnSO}_{4}$ & 1.00 \\
$\mathrm{~K}_{2} \mathrm{SO}_{4}$ & 0.30 & $\mathrm{ZnSO}_{4}$ & 0.67 \\
$\mathrm{MgSO}_{4}$ & 0.67 & $\mathrm{CuSO}_{4}$ & 0.13 \\
$\mathrm{CaCl}_{2}$ & 0.23 & $\left(\mathrm{NH}_{4}\right)_{6} \mathrm{Mo}_{7} \mathrm{O}_{24}$ & 0.047 \\
$\mathrm{Al}_{2}\left(\mathrm{SO}_{4}\right)_{3}$ & 0.035 & & \\
\hline
\end{tabular}

Table S2. Spiked levels, recoveries, relative standard deviations (RSDs).

\begin{tabular}{cccc} 
matrix & $\begin{array}{c}\text { spiked level } \\
(\mathrm{mg} / \mathrm{kg})\end{array}$ & $\begin{array}{c}\text { Mean recovery } \\
(\%, \mathrm{n}=5)\end{array}$ & $\begin{array}{c}\text { RSD } \\
(\%, \mathrm{n}=5)\end{array}$ \\
\hline $\begin{array}{c}\text { Cell walls } \\
(\mathrm{F} 1)\end{array}$ & 0.004 & 87.9 & 8.0 \\
& 0.1 & 86.0 & 7.5 \\
& 0.5 & 84.0 & 5.6 \\
Organelles & 5 & 92.4 & 3.4 \\
$(\mathrm{~F} 2)$ & 0.004 & 98.6 & 15.1 \\
& 0.1 & 97.0 & 2.9 \\
& 0.5 & 83.4 & 8.8 \\
& 5 & 108.8 & 3.1 \\
Soluble & 0.01 & 92.0 & 6.1 \\
fractions (F3) & 1 & 104.9 & 4.9 \\
& 10 & 100.3 & 6.8 \\
\hline
\end{tabular}

Table S3. Linear regression, $\mathrm{R}^{2}, \mathrm{MEs}$.

\begin{tabular}{ccccc}
\hline Matrix & $\begin{array}{c}\text { Linearity range } \\
(\mathrm{mg} / \mathrm{L})\end{array}$ & Regression eq & $\mathrm{R}^{2}$ & $\mathrm{MEs}$ \\
\hline Solvent & $0.005-1$ & $\mathrm{y}=195.2 \mathrm{x}-0.200$ & 0.9999 & - \\
Cell walls & $0.005-1$ & $\mathrm{y}=143.5 \mathrm{x}-0.127$ & 0.9997 & 0.74 \\
Cell organelles & $0.005-1$ & $\mathrm{y}=144.7 \mathrm{x}+1.094$ & 0.9971 & 0.74 \\
Soluble fractions & $0.005-1$ & $\mathrm{y}=219.2 \mathrm{x}+0.521$ & 0.9998 & 1.12 \\
\hline
\end{tabular}


Table S4. Linear regression of tissue perchlorate concentration (21st day) and exposure concentration.

\begin{tabular}{cccc}
\hline Cultivar & Tissue & Regression eq & $\mathrm{R}^{2}$ \\
\hline Fuding Dabai & Tender leaf & $\mathrm{y}=3.508 \mathrm{x}-0.308$ & 0.9995 \\
& Mature leaf & $\mathrm{y}=3.166 \mathrm{x}+0.168$ & 0.9999 \\
Wuniuzao & Root & $\mathrm{y}=0.405 \mathrm{x}+0.064$ & 0.9997 \\
& Tender leaf & $\mathrm{y}=3.846 \mathrm{x}+1.340$ & 0.9967 \\
& Mature leaf & $\mathrm{y}=3.052 \mathrm{x}+1.175$ & 0.9978 \\
\hline
\end{tabular}

Table S5. The bioconcentration factor (BCF) of field samples.

\begin{tabular}{|c|c|c|c|}
\hline Province & $\begin{array}{c}\text { Soil } \\
(\mathrm{mg} / \mathrm{kg})\end{array}$ & $\begin{array}{c}\text { Fresh leaves } \\
(\mathrm{mg} / \mathrm{kg})\end{array}$ & $\mathrm{BCF}$ \\
\hline \multirow[t]{16}{*}{ Zhejiang } & 0.041 & 0.912 & 22.50 \\
\hline & 0.036 & 0.077 & 2.12 \\
\hline & 0.008 & 0.016 & 1.88 \\
\hline & 0.013 & 0.034 & 2.52 \\
\hline & 0.009 & 0.018 & 1.88 \\
\hline & 0.022 & 0.947 & 43.82 \\
\hline & 0.007 & 0.012 & 1.73 \\
\hline & 0.008 & 0.209 & 24.75 \\
\hline & 0.009 & 0.199 & 21.22 \\
\hline & 0.011 & 0.133 & 12.41 \\
\hline & 0.019 & 0.081 & 4.37 \\
\hline & 0.007 & 0.147 & 22.13 \\
\hline & 0.004 & 0.019 & 4.87 \\
\hline & 0.014 & 0.035 & 2.50 \\
\hline & 0.001 & 0.034 & 24.29 \\
\hline & 0.004 & 0.036 & 10.29 \\
\hline \multirow[t]{2}{*}{ Yunnan } & 0.013 & 0.001 & 0.10 \\
\hline & 0.013 & 0.007 & 0.52 \\
\hline \multirow[t]{8}{*}{ Hunan } & 0.028 & 0.225 & 7.94 \\
\hline & 0.019 & 0.104 & 5.42 \\
\hline & 0.021 & 0.109 & 5.14 \\
\hline & 0.020 & 0.094 & 4.64 \\
\hline & 0.020 & 0.174 & 8.58 \\
\hline & 0.020 & 0.063 & 3.11 \\
\hline & 0.020 & 0.125 & 6.17 \\
\hline & 0.020 & 0.056 & 2.79 \\
\hline
\end{tabular}




\begin{tabular}{cccc} 
& 0.020 & 0.088 & 4.39 \\
\hline Fujian & 0.016 & 0.108 & 6.90 \\
& 0.016 & 0.260 & 16.59 \\
0.016 & 0.083 & 5.31 \\
0.015 & 0.569 & 38.29 \\
0.010 & 0.233 & 23.92 \\
0.010 & 0.204 & 20.94 \\
0.010 & 0.194 & 19.96 \\
& 0.007 & 0.195 & 28.03 \\
0.007 & 0.232 & 33.41 \\
& 0.006 & 0.161 & 26.22 \\
0.006 & 0.170 & 27.59 \\
0.006 & 0.238 & 38.63 \\
0.006 & 0.227 & 36.92 \\
0.006 & 0.144 & 23.32 \\
0.006 & 0.250 & 40.53 \\
\hline
\end{tabular}

Table S6. The BCFs of perchlorate in plant leaves.

\begin{tabular}{ccc}
\hline Plant & $\begin{array}{c}\text { Exposure concentration } \\
(\mathrm{mg} / \mathrm{L})\end{array}$ & $\mathrm{BCF}$ \\
\hline Snap bean & $0.01-10$ & $55-70$ \\
Willow & 10 & 10 \\
& 20 & 5.7 \\
Smartweed & 0.1 & 8.2 \\
Lettuce & $1-2$ & $4.8-13.2$ \\
& $0.001-0.01$ & $2-40$ \\
& $0.026-0.181$ & $62-292$ \\
\hline
\end{tabular}




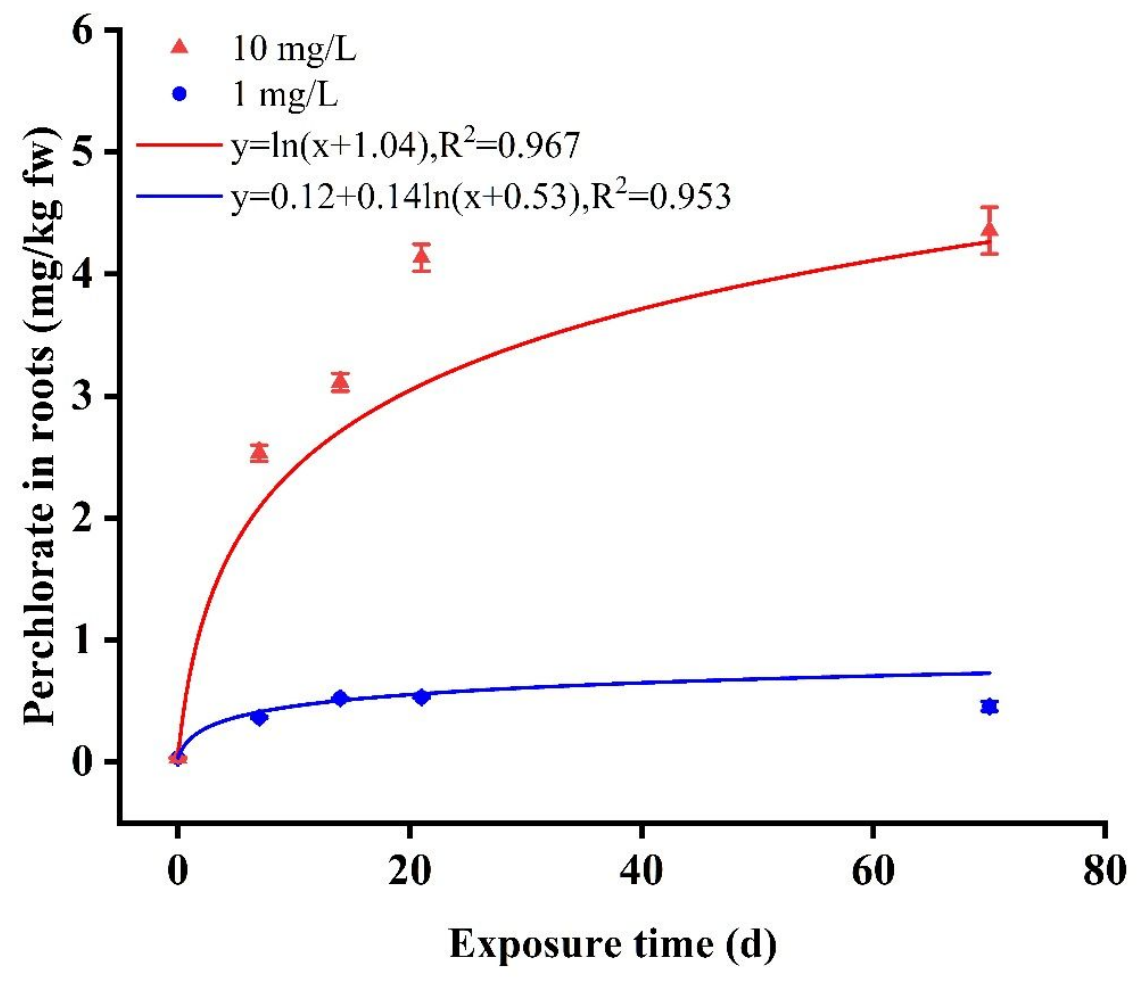

Figure S1. Perchlorate concentration in root of Wuniuzao under 1 and $10 \mathrm{mg} / \mathrm{L}$ perchlorate treatment as a function of exposure time. $(n=3)$ 

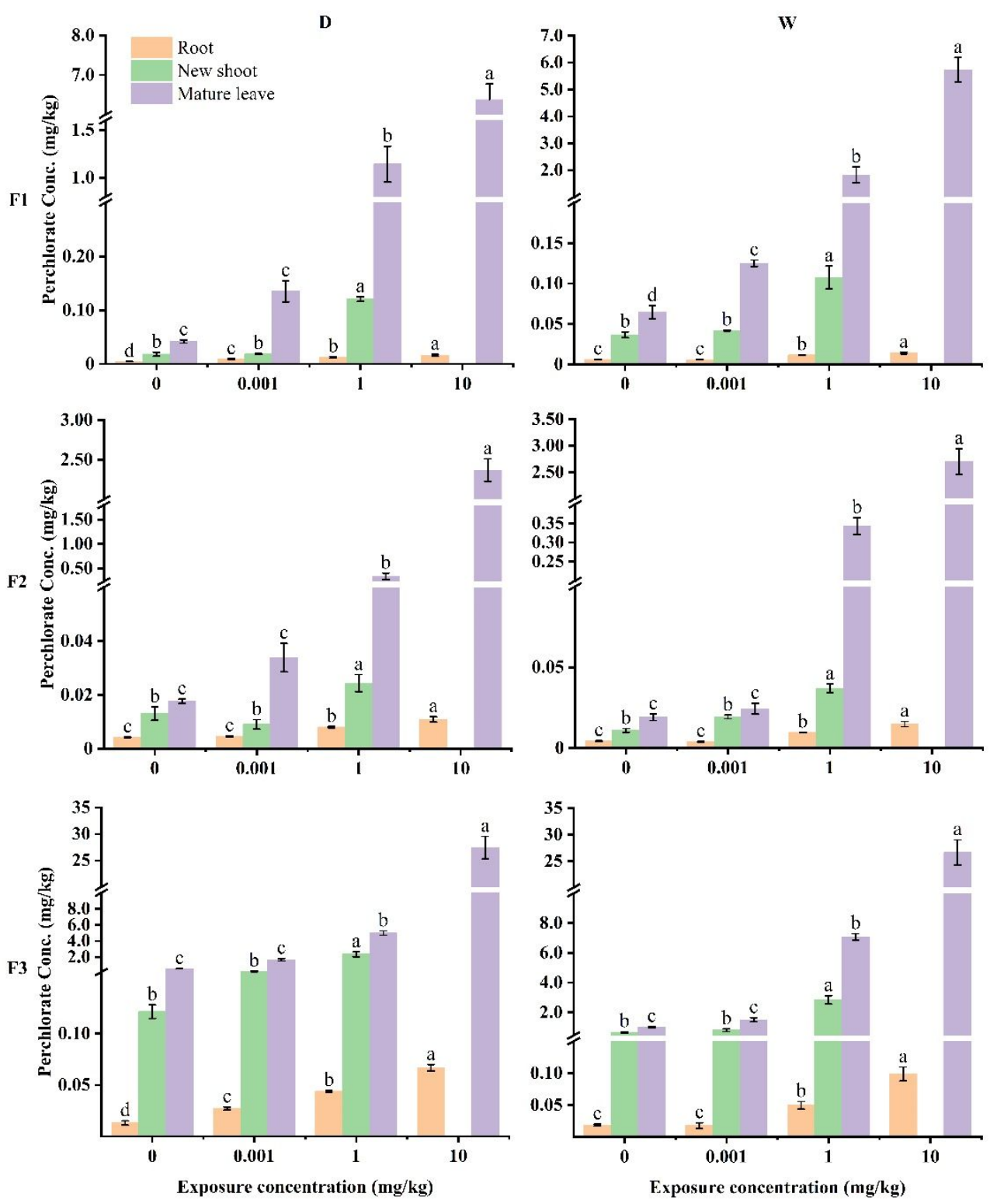

Figure S2. Perchlorate concentration of different tissues in each subcellular fraction. Roots(R), new shoots(B), mature leaves(M). Fuding Dabaicha(D), Wuniuzao(W). Subcellular fraction: cell wall(F1), cell organelles(F2) and soluble fractions(F3), (n=3). Different letters indicate significant differences $(p<0.05)$ among exposure concentrations. 